\title{
Approvisionnement en eau et risques sanitaires dans le bassin versant amont de l'Abiergué à Yaoundé (Cameroun)
}

\author{
Emmanuel Kengmoe Tchouongsi, \\ Benoît Mougoue, \\ Claude Tatuebu Tagne, \\ Fabrice Mopi Touoyem, \\ Ngoran Simplice Bonganjum,
} Département de Géographie, Université de Yaoundé 1, Cameroun

\section{Résumé}

La santé des populations dépend de la qualité de l'eau et des aliments qu'elles consomment. En milieu urbain, notamment dans les quartiers précaires des pays en développement où l'accès à la ressource en eau demeure difficile, les eaux consommées ne sont pas toujours de bonne qualité à cause de leur contamination par diverses sources de pollution. Cette situation expose les populations aux maladies hydriques. L'objectif de cet article est de montrer l'impact des différents modes d'approvisionnement en eau sur la santé des populations du bassin versant amont de l'abiergué. La méthodologie adoptée était basée sur la revue de littérature, l'observation participante, les entretiens semi-structurés, les enquêtes par questionnaires puis les analyses physicochimiques et bactériologiques. La recherche documentaire a consisté à collecter un certain nombre de données qualitatives sur le sujet en consultant divers documents sur internet et certains ouvrages (mémoires, thèses, etc.) sur cette thématique. Par ailleurs, les autres méthodes utilisées, nous ont permis de collecter des données quantitatives et qualitatives sur les modes d'approvisionnement en eau, l'usage des ressources en eau, la gestion des eaux usées et des déchets, etc. En parallèle, des analyses physico-chimiques et bactériologiques ont été effectuées dans trois types d'ouvrages d'approvisionnement en eau. Les résultats obtenus indiquent que les populations des quartiers étudiés s'approvisionnent essentiellement au niveau des puits $(n=136)$, sources $(n=7)$ et bornes fontaines $(n=8)$ se trouvant à proximité des latrines, des ordures ménagères et des eaux usées susceptibles de les polluer. Les paramètres physico-chimiques $(\mathrm{pH}$, nitrate, température et conductivité) des eaux prélevées dans la zone d'étude ne respectent pas 
totalement les normes camerounaises, françaises et de l'OMS relatives à la potabilité de l'eau. De plus, les résultats des analyses bactériologiques montrent une concentration élevée des coliformes fécaux dans les eaux collectées (237 UFC/100 ml pour les eaux du puits sommaire de Melen 7A ; $57 \mathrm{UFC} / 100 \mathrm{ml}$ pour les eaux du puits amélioré de Melen 9 ; $38 \mathrm{UFC} / 100 \mathrm{ml}$ pour les eaux de la source aménagée de Melen 6). De même, les résultats obtenus révèlent une forte concentration des streptocoques fécaux dans les eaux de la zone d'étude (11 UFC/100 ml pour les eaux de source aménagée de Melen $6 ; 6 \mathrm{UFC} / 100 \mathrm{ml}$ pour les eaux du puits sommaire de Melen 7A et 9 UFC/100 ml pour les eaux du puits amélioré de Melen 9). Au regard de ces résultats, il ressort que les eaux de la zone d'étude sont impropres à la consommation et susceptibles de nuire à la santé humaine.

Mots-clés : Approvisionnement en eau, risque sanitaire, bassin versant, Abiergué, Yaoundé

\title{
Water provision and Health risks in the upper drainage basin of Abiergué in Yaoundé (Cameroon)
}

\author{
Emmanuel Kengmoe Tchouongsi, \\ Benoît Mougoue, \\ Claude Tatuebu Tagne, \\ Fabrice Mopi Touoyem, \\ Ngoran Simplice Bonganjum,
}

Département de Géographie, Université de Yaoundé 1, Cameroun

\begin{abstract}
The health of the population depends on water quality and food they consume. Dwellers living in slums of urban areas in developing countries have difficult access to water resources. Water is contaminated by diverse sources of pollution which reduces its quality. This situation exposes the population to waterborne diseases. The objective of this article is to show case the impacts of different water provision modes on the health of the population in the upstream of Abiergué drainage basin. The literature review, participatory observation, semi structured interviews, questionnaire survey, physicochemical and bacterial analysis constituted the methodological base of this study. The documentary research permitted this study to obtain qualitative data from diverse documents consulted (memoires, theses, etc). Other methods
\end{abstract}


utilized permitted this study to collect quantitative and qualitative data on different modes of water provision, water usages, management of waste and used water. Moreover, physico-chemical analysis were carried out on three different water provision sources. The results obtained showed that the population of study districts, are essentially supplied by wells $(n=136)$, springs $(n=7)$, bore holes $(n=8)$ that are closer to toilets, household waste and used water susceptible of polluting them. Physico-chemical parameters ( $\mathrm{pH}$, nitrate, temperature and conductivity) of water samples taken from the study area show that they do not respect Cameroonian, French, and WHO norms on potable water. Bacterial analysis attest that there is high concentration of fecal coliforms collected (237 UFC/100 ml for rudimentary wells of Melen 7A; 57 $\mathrm{UFC} / 100 \mathrm{ml}$ for ameliorated wells of Melen 9; $38 \mathrm{UFC} / 100 \mathrm{ml}$ for good springs of Melen 6).In the same vain, the results obtained shows a high concentration of fecal streptococcus in waters of the study area (11UFC/100 $\mathrm{ml}$ for good springs of Melen 6; $6 \mathrm{UFC} / 100$ for rudimentary wells of Melen 7A and $9 \mathrm{UFC} / 100 \mathrm{ml}$ for ameliorated wells of Melen 9). Conclusively, these results show that water sources found in the study area are not good for consumption and susceptible to cause health problems.

Keywords: Water supply, health risk, watershed, Abiergué, Yaoundé

\section{Introduction}

Dans les zones urbaines des pays en développement, l'accès à une eau de qualité est un calvaire pour de milliers de citadins. Si l'on a très souvent coutume d'affirmer que « l'eau c'est la vie », cela relève tout simplement du fait que la consommation d'une eau de qualité est indispensable pour la bonne santé et la survie des êtres vivants (Plan international, 2015).

La conférence internationale sur l'eau et l'environnement tenue à Dublin en 1992 a réaffirmé le caractère vital de l'eau dans son principe premier indiquant que "l'eau douce, ressource fragile et non renouvelable, est indispensable à la vie, au développement et à l'environnement » (Déclaration de Dublin, 1992). L'accès à l'eau salubre et à l'assainissement constitue également l'un des défis majeurs poursuivis par les Objectifs du Développement Durable (ODD) fixés par l'Organisations des Nations Unies (ONU, 2015).

Avec une population estimée aujourd'hui à environ 2500000 habitants et une croissance urbaine sans précédent, la ville de Yaoundé fait face à des pénuries d'eau (PDUY, 2015). Selon les chiffres de la Camerounaise des eaux, en 2011, seuls $10000 \mathrm{~m}^{3}$ d'eau étaient disponibles pour les populations alors que la demande globale en eau s'élevait à 250000 $\mathrm{m}^{3}$ d'eau par jour. 
Outre, ces insuffisances en termes de fourniture en eau, la mauvaise qualité de l'eau servie aux populations et son faible débit au niveau des robinets constituent d'énormes problèmes au niveau de nombreux ménages de la capitale camerounaise. D'après les informations recueillies auprès des populations, les habitants de certains quartiers de la ville font plusieurs jours, plusieurs mois et parfois des années sans eau potable.

De même, les constats faits sur le terrain indiquent que les habitants du bassin versant amont de l'Abiergué utilisent divers points d'eau, plus ou moins aménagés (puits, forages et source) pour se ravitailler en eau. Les populations qui utilisent ces eaux souvent contaminées par les latrines et les eaux usées, se plaignent de diverses maladies dont les infections cutanées et/ou les maladies d'origine hydrique. Ngnikam et al. (2007) ont relevé la persistance chez des milliers de citadins des zones sous-intégrées, des pathologies dues au caractère non potable de l'eau de consommation, notamment le choléra, la dysenterie amibienne, les infections parasitaires intestinales. Le but de cette étude est d'analyser l'impact de la qualité des eaux de consommation sur la santé des habitants du bassin versant de l'Abiergue à Yaoundé.

\section{Matériel et méthodes}

\subsection{Zone d'étude : bassin versant amont de l'Abiergué}

Situé dans la Commune d'Arrondissement de Yaoundé $6^{\text {ème }}$, le bassin versant amont de l'Abiergué s'étend sur 5 quartiers, à savoir Nkolbikok 2, Melen 6, Melen 7B, Melen 7A et Melen 9 (PCD, 2013). Ce sont ces 5 quartiers qui ont constitué les sites d'étude de ce travail.

Le bassin versant amont de l'Abiergué couvre près de 72 hectares et abrite environ 15000 habitants (BUCREP, 2005). Au plan géographique, il est limité au nord-ouest par les quartiers Nkolbikok I et la Cité Verte, au sudouest par le quartier Mvog Bétsi, au nord-est par les quartiers Nkolbikok, EligEffa et Melen4, et au sud-est par le quartier Mvog Betsi (Figure 1)

La ville de Yaoundé subit l'influence du climat tropical humide de transition caractérisé par une pluviométrie bimodale avec quatre saisons bien marquées (Suchel, 1987):

- une grande saison de pluies qui va de mars à juin ;

- une courte saison de pluies qui dure de septembre à octobre mais plus importante en intensité ;

- une grande saison sèche allant de décembre à février ;

- une petite saison sèche qui s'étend de juillet à août. 


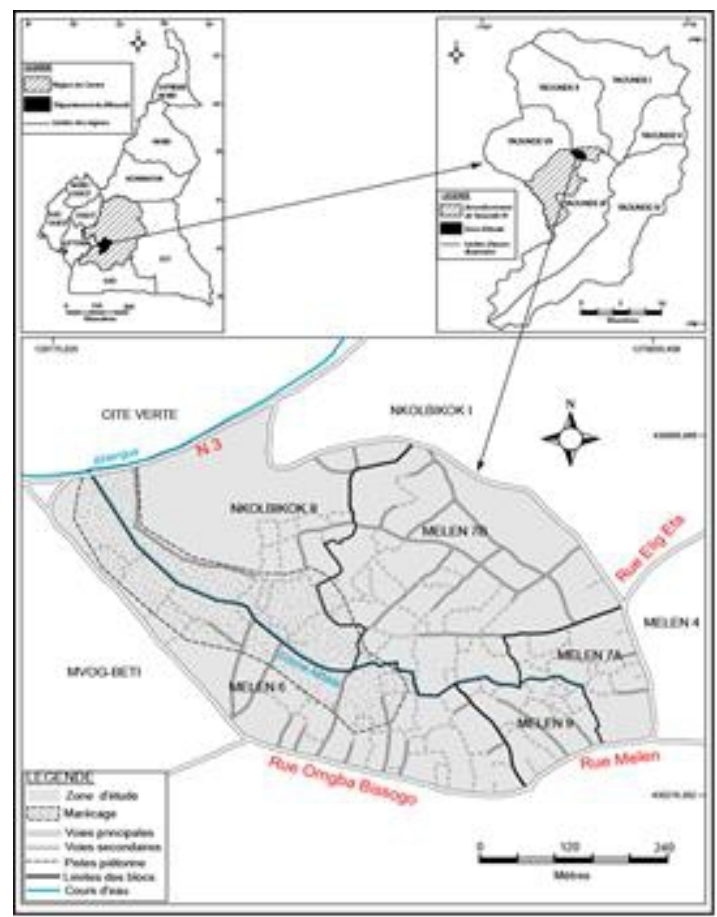

Figure 1 : Localisation géographique du bassin versant amont de l'Abiergué (Source : Fond de carte de la Communauté Urbaine de Yaoundé 2012)

La température moyenne annuelle tourne autour de $23,5^{\circ} \mathrm{C}$ et la pluviométrie moyenne annuelle est d'environ $1565 \mathrm{~mm}$ (Suchel, 1987). Pendant la courte saison de pluies, les populations des bas-fonds du basin versant amont de l'Abiergué font face à de nombreux problèmes d'inondation à cause de la montée des eaux de la rivière Edjoa Mballa. Et c'est au cours de cette même période que les latrines à canon sont vidangées.

Au plan topographique, la zone d'étude est très accidentée et présente des interfluves séparés par des vallées marécageuses (Figure 2). D'une altitude moyenne de $742 \mathrm{~m}$, les zones les plus basses de la zone étudiée se situent à $715 \mathrm{~m}$ d'altitude tandis que celles les plus hautes se trouvent à $745 \mathrm{~m}$. 


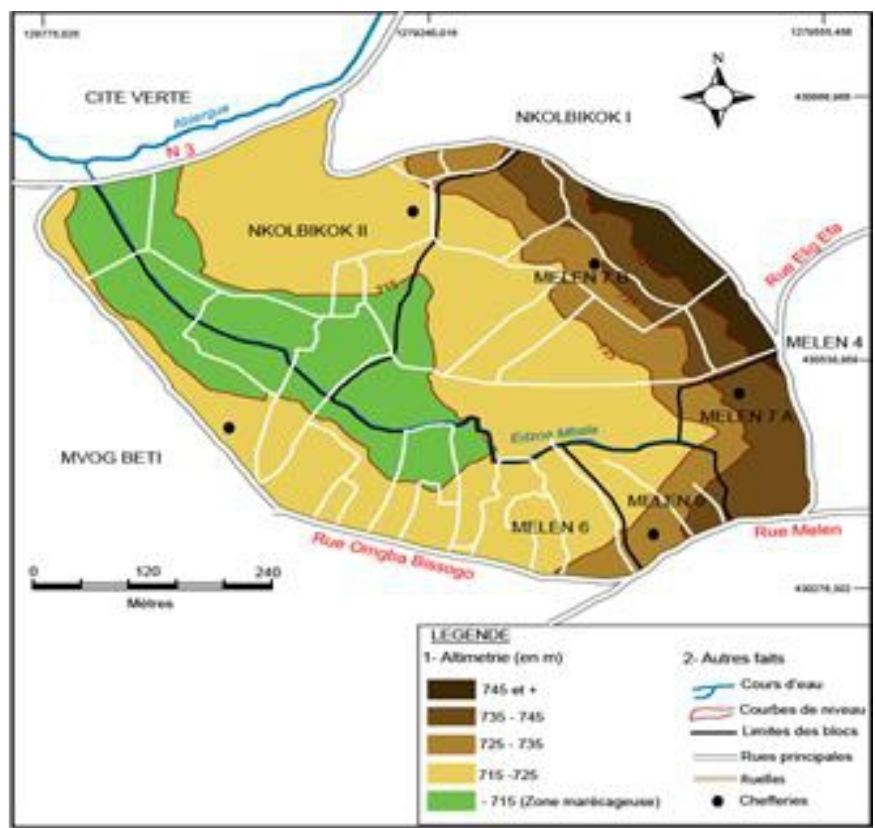

Figure 2: Altimétrie du bassin versant amont de l'Abiergué

(Source : Modèle Numérique de Terrain 2013)

Les zones de faibles pentes où sont entassées les populations humaines sont souvent le siège d'inondations et de stagnation des eaux (Sanctoir et Bopda, 1995). D’une manière générale, l'étagement du relief de la zone d'étude permet de distinguer les zones de fortes pentes, les zones de moyennes pentes et les zones de faibles pentes (Figure 2).

Son réseau hydrographique est riche et dense. Les principaux cours d'eaux qui drainent ce territoire sont les rivières Edjoa Mballa et Abiergué (PCD, 2013). Les observations faites sur le terrain ont permis de relever que, le réseau hydrographique collecte les eaux de ruissellement et assurent l'assainissement de la zone d'étude. En général, les cours d'eau servent d'exutoires naturels à tous les déchets générés par les habitants. L'écoulement des eaux usées épouse la topographie. Dans les bas-fonds, plusieurs toilettes à canon et sur pilotis sont construites le long des cours d'eau et des drains naturels. En période de crues, la rivière Edjoa Mballa déborde de son lit et charrie tout ce qu'elle trouve à son passage.

L'analyse de l'occupation de l'espace à partir de l'image google earth (Figure 3) montre que le bassin versant amont de l'Abiergué se détache nettement des autres quartiers non seulement par son ancienneté et sa forte densité démographique, mais surtout par un entassement anarchique du bâti. La situation est de plus en plus inextricable compte tenu de la croissance démographique qui reste constante et l'occupation anarchique sans cesse grandissante dans cette zone. Globalement sur le terrain, il y a une forte 
concentration de l'habitat depuis les bas de pente jusqu'au sommet, et un relâchement au niveau des interfluves.

Cette zone du bassin versant est habitée par des personnes essentiellement pauvres (ECAM, 2016). Les visites de terrain ont permis de constater que les maisons y sont de nature hétéroclite avec une prédominance des latrines sommaires et généralement à canon. Les maisons en planches de récupération dominent le secteur. Les inondations surviennent régulièrement en saison de pluies dans ce quartier à habitat précaire. Les rigoles concourant à l'évacuation des eaux usées sont souvent obstruées par les ordures de diverses natures.

\subsection{Collecte des données}

La démarche méthodologique retenue a nécessité l'utilisation de la recherche documentaire, les entretiens semi-directifs, les observations de terrain, les enquêtes, puis les analyses physico-chimiques et bactériologiques des échantillons d'eau.

Les entretiens semi-directifs avec les acteurs de l'assainissement travaillant au niveau de ce bassin versant ont été réalisés. Cela a permis de mieux comprendre leurs logiques et les stratégies d'action en matière de gestion des ordures ménagères, des eaux usées et d'approvisionnement des habitants en eau. De plus, des observations directes ont été faites dans les cinq (5) quartiers d'étude. Cette technique a été très utile dans la perception des formes d'assainissement et des modes d'approvisionnement en eau, l'appréciation de l'état des infrastructures d'assainissement (latrines, fosses septiques, canaux d'évacuation, etc.) et de l'insalubrité dans les différents quartiers. Par la suite, des questionnaires ont été administrés à 357 ménages riverains de la zone d'étude, répartis en trois strates: la zone d'habitat structuré (STRATE A), la zone d'habitat spontané (STRATE B) et la zone marécageuse (STRATE C) (Figure 3). 


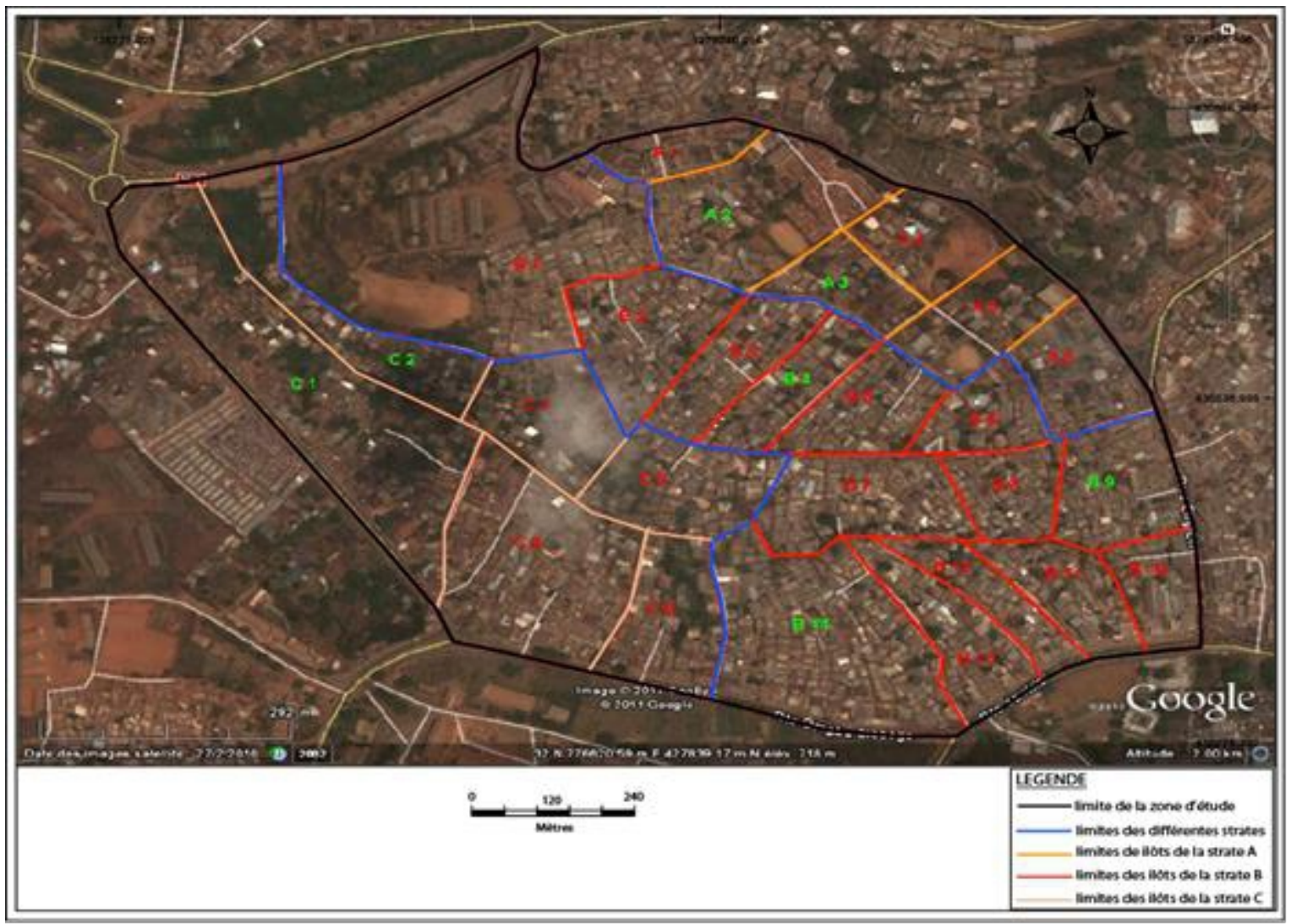

Figure 3: Représentation des strates et îlots dans la zone d'étude (Source : Google Earth, 2012)

En parallèle, des échantillons d'eau ont été prélevés dans les 5 sites d'étude le 09 juillet 2013 entre $8 \mathrm{~h}$ et $9 \mathrm{~h}$. Le choix de ces points d'échantillonnage s'est basé sur les caractéristiques urbanistiques du quartier, le degré d'aménagement des puits, la distance du puits aux latrines les plus proches, le nombre de latrines autour du puits, l'existence d'autres sources de pollution autour du puits, le taux de fréquentation du puits, et l'usage de l'eau par les populations humaines.

A cet effet, le présent travail s'est focalisé sur trois (3) différentes sources d'approvisionnement en eau préférées par les populations, à savoir : les puits sommaires, les puits améliorés et les sources aménagées. Par la suite, les échantillons d'eau prélevés ont été conditionnés dans des glacières puis acheminés au laboratoire en vue des analyses physico-chimiques (température, nitrates, conductivité, $\mathrm{pH}$ ) et bactériologiques (streptocoques fécaux et coliformes fécaux).

La température de l'eau a été déterminée in situ à l'aide d'un conductimètre de marque HQ14d. Par contre, le $\mathrm{pH}$ a été déterminé par lecture directe sur un pH-mètre de marque Hach (HQ11d) (Kengmoé, 2013). 
La conductivité électrique exprimée en $(\mu \mathrm{S} / \mathrm{cm})$ a été mesurée sur le terrain en utilisant un conductimètre de marque Hach (HQ14d).

Enfin, les quantités d'ions nitrates $\left(\mathrm{NO}_{3}{ }^{-}(\mathrm{mg} / \mathrm{l})\right.$, ont été évaluées par la méthode de réduction au cadmium via un spectrophotomètre de marque Hach DR/2010 ; les différentes valeurs ont été exprimées en mg/l (Kengmoé, 2013).

Les analyses bactériologiques des eaux ont porté uniquement sur la recherche des germes indicateurs de la pollution fécale, notamment les coliformes fécaux et les streptocoques fécaux (Kengmoé, 2013). Pour le dénombrement des coliformes fécaux, la méthode d'analyse par «colorimétrie sur membrane filtrante » a été utilisée.

Quant à la quantification des streptocoques fécaux, elle s'est basée sur la méthode de «filtration sur membranes » (Kengmoé, 2013).

Nota bene: Toutes les analyses bactériologiques ont été réalisées à partir des prélèvements faits pendant la campagne de saison des pluies.

\subsection{Analyses des données}

Les données collectées à partir des méthodes des sciences sociales ont fait l'objet d'un d'une saisie et d'un traitement graphique dans le tableur Excel version 2010. Par contre, les coordonnées cartographiques des latrines et des fosses septiques, des points d'approvisionnement en eau et celles des zones de rejet des eaux usées et de dépôt des ordures ménagères ont été importées et intégrées dans le logiciel ArcGis 10.4 afin de spatialiser les différents phénomènes et de décrire les variables étudiées.

Pour la quantification des coliformes fécaux, les colonies ont été dénombrées au laboratoire Waste Water Reasearch Unit de l'Université de Yaoundé I, après incubation et leur nombre total a été estimé à partir de la formule ci-dessous :

$$
\mathrm{UFC}=\frac{\text { Nombre de colonies comptées }}{\text { Volume d'échantillon filtré }(\mathrm{ml})} \times 100 \mathrm{ml}
$$

Où UFC = Unité Formatrice de Colonie pour $100 \mathrm{ml}$.

De plus, pour évaluer la quantité des streptocoques fécaux, les colonies ont été dénombrées dans le même laboratoire et leur nombre total a été estimé en utilisant la formule de calcul des coliformes fécaux susmentionnée.

\section{Résultats}

2.1. Modes d'approvisionnement en eau dans le bassin versant amont de l'Abiergué

L'approvisionnement en eau des populations du bassin versant amont de l'Abiergué se fait globalement de deux manières, soit à travers le réseau 
d'adduction d'eau potable de la Camwater, soit via les solutions décentralisées d'approvisionnement en eau, à savoir les bornes fontaines publiques, les puits et les sources d'eau.

\subsubsection{Réseau de la Camwater}

Le réseau d'adduction d'eau potable n'existe que dans la partie lotie du site (versants, sommets et interfluves). Ce réseau ne permet pas à toute la population de cette zone d'avoir accès à l'eau potable. Les problèmes d'accès à l'eau potable sont principalement liés au coût de connexion au réseau d'adduction d'eau qui varie en fonction de l'éloignement des ménages demandeurs. De plus, la promiscuité qui y règne et qui entrave le passage des différents conduits, favorise aussi ces difficultés d'approvisionnement en eau potable. En conséquence, la majorité des familles n'est pas connectée au réseau de la Camwater.

Pour faire face à ce déficit en eau de la Camwater, les populations lésées utilisent essentiellement l'eau issue des points d'approvisionnement décentralisés tels que les Bornes Fontaines Payantes (BFP), les puits et les sources d'eau.

\subsubsection{Bornes Fontaines Payantes}

Les ménages non abonnés à la Camwater s'approvisionnent au niveau des Bornes Fontaines Payantes (BFP). Dans le bassin versant amont de l'Abiergué, on dénombre seulement 8 Bornes Fontaines Payantes (Tableau 1). Ces ouvrages hydrauliques sont pour la plupart installés en bordure des quartiers.

Tableau 1: Répartition des bornes fontaines payantes dans les quartiers d'étude

\begin{tabular}{|c|c|c|}
\hline $\mathbf{N}^{\circ}$ & Quartiers d'étude & Nombre de BFP \\
\hline $\mathbf{1}$ & Nkolbikok II & 2 \\
\hline $\mathbf{2}$ & Melen 7B & 3 \\
\hline $\mathbf{3}$ & Melen 7 A & 1 \\
\hline $\mathbf{4}$ & Melen 6 & 2 \\
\hline $\mathbf{5}$ & Melen 9 & 0 \\
\hline & Total & $\mathbf{8}$ \\
\hline
\end{tabular}

Source : Enquêtes de terrain (2011)

\subsubsection{Puits}

Dans le bassin versant amont de l'Abiergué, les populations ne disposant pas d'un branchement sur le réseau d'eau de la Camwater utilisent essentiellement trois (3) types de puits : les Puits Non Aménagés, les Puits Sommairement Aménagés et les Puits Aménagés. 


\section{Les Puits Non Amenagés (PNA)}

Les PNA sont des puits non aménagés ne disposent d'aucun élément de sécurité, ni de protection de la ressource en eau. Leur environnement immédiat n'est ni drainé, ni bétonné (Photo 1). Dans la zone d'étude, ils sont au nombre de 72 , soit $52,94 \%$.

Aussi, les risques d'infection des usagers, de contamination de la ressource en eau par les latrines voisines, par les ordures ménagères et par les eaux usées stagnantes, sont probablement élevés. L'eau issue des Puits Non Aménagés (Photo 1) est utilisée essentiellement pour les travaux ménagers (vaisselle, lessive, cuisine, propreté de la maison, etc.) et très rarement pour la consommation humaine. Ce type de puits est très répandu dans la zone étudiée $(\mathrm{n}=72)$.

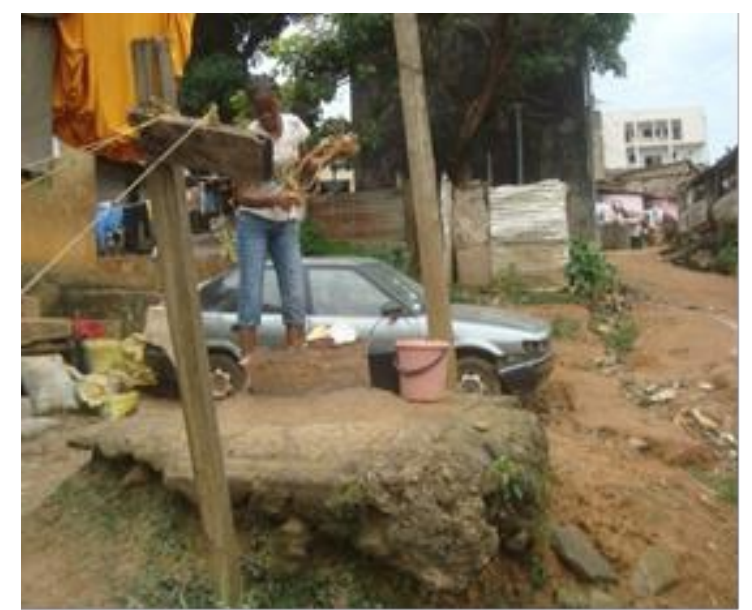

Photo 1: Puits non aménagé en plein air à Nkolbikok 2

(Cliché Kengmoé, 2013)

\section{Les Puits Sommairement Aménagés (PSA)}

S'agissant des puits sommairement aménagés, ce sont des ouvrages présentant des débuts d'aménagement dans le but d'assurer relativement la sécurité des usagers lors du puisage et la protection de la ressource hydraulique. La voie d'accès des usagers à ces ouvrages est relativement de bonne qualité, mais, elle est parfois glissante en saison des pluies. Bien que les risques de pollution de la ressource en eau soient importants avec la proximité des latrines à fonds perdus, des eaux usées stagnantes et des dépôts anarchiques d'ordures ménagères, l'eau prélevée dans ces PSA (Photo 2) est utilisée par les habitants pour les travaux domestiques. Mais, dans certaines endroits, cette eau est utilisée comme eau de cuisson et de boisson. On dénombre une multitude de ces ouvrages dans le bassin versant amont de l'Abiergué $(\mathrm{n}=52)$. 


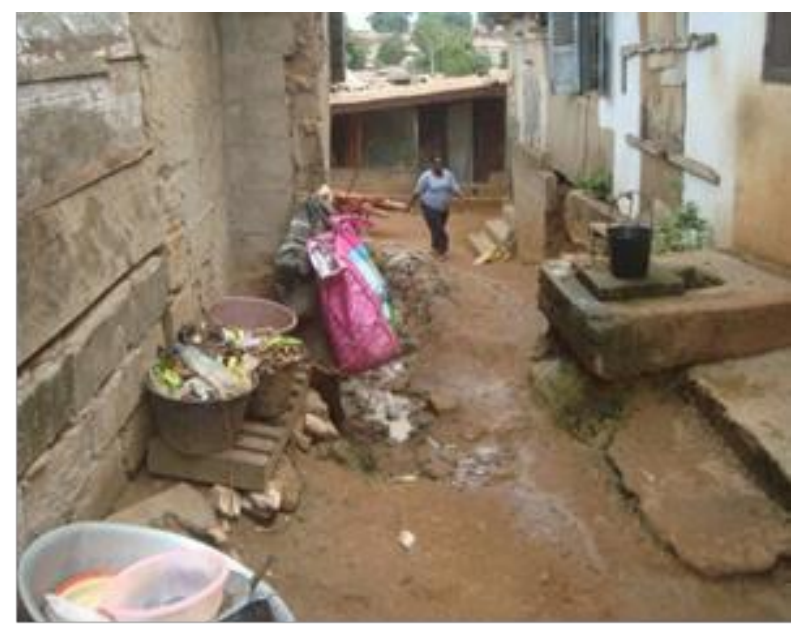

Photo 2 : Puits sommairement aménagé à Melen 9

(Cliché Kengmoé, Mai 2012)

\section{Les Puits Aménagés}

Les Puits Aménagés (PA) sont des ouvrages construits selon les règles de l'art et les normes (Photo 3). Ils sont équipés de dispositifs de sécurité, de confort, d'hygiène et de protection de la ressource en eau (Photo 3). L'eau issue de ce type d'ouvrage est généralement destinée à l'usage domestique et à la consommation. Dans la zone d'étude, 12 puits aménagés ont été identifiés.

En général, dans le bassin versant amont de l'Abiergué, 68,90\% des ménages ont recours à l'eau des puits, alors que 31,09\% n'utilisent pas l'eau provenant de ces ouvrages.

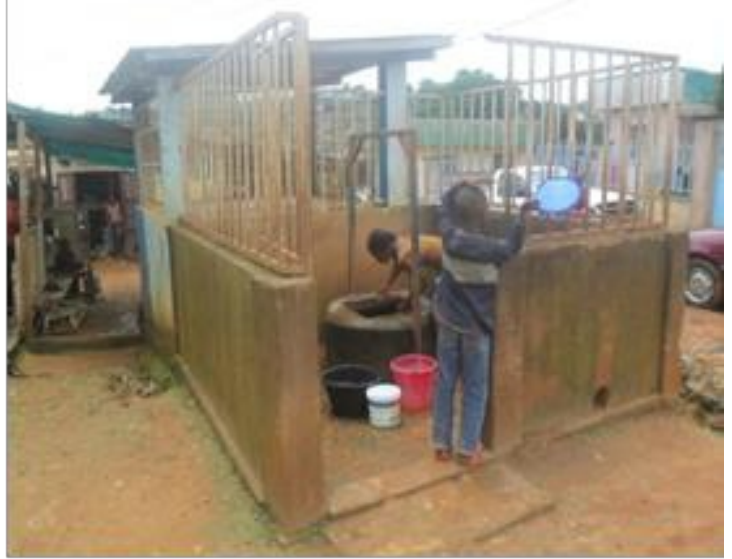

Photo 3: Puits aménagé à Melen

(Cliché Kengmoé, Mai 2012)

\subsubsection{Sources d'eau}

Dans le bassin versant amont de l'Abiergué, près de 13,16\% des ménages utilisent l'eau de source alors que $86,83 \%$ ne l'utilisent pas. 
Il existe plusieurs sources d'eau dans les quartiers de notre zone d'étude. Sur la base de la classification proposée par Djeuda (2001), on peut distinguer : les Sources Aménagées et les Sources Non Aménagées.

\section{Les Sources Aménagées}

Il s'agit d'ouvrages ayant bénéficié d'un aménagement autour du point de résurgence de la nappe souterraine. Certains de ces ouvrages sont équipés de conduits de puisage en PVC de diamètre compris entre 25 et $40 \mathrm{~cm}$. Cependant, ces sources sont souvent polluées par les latrines, les eaux usées et les puits avoisinants (Photo 4).

\section{Les Sources Non Aménagées}

Ce sont des points de captage d'eau souterraine en forme de cuvette. Ces sources ne possèdent ni couvercle, ni dispositif d'étanchéité, encore moins de filage (Photo 5). On rencontre généralement ce type d'ouvrage dans les zones marécageuses. La rétention du filet d'eau est assurée par la présence d'un sol argileux. L'eau recueillie dans ce type d'ouvrage sert généralement aux travaux ménagers, et parfois, d'eau de boisson. Dans le bassin versant amont de l'Abiergué, ces sources d'eau sont polluées par les eaux vannes issues des latrines, des eaux usées domestiques et des ordures ménagères (Photo 5).

En revanche, le manque d'entretien de ces points d'eau et l'absence d'assainissement dans les différents quartiers de la zone d'étude (stagnation des eaux usées, mauvaise gestion des déchets ménagers, vidange des latrines dans les rivières et construction des latrines près des puits et des sources d'eau) augmentent les risques de contamination des eaux utilisées par les populations riveraines. Cet état de fait les expose ainsi à diverses pathologies dont les plus importantes sont les maladies d'origine hydrique.

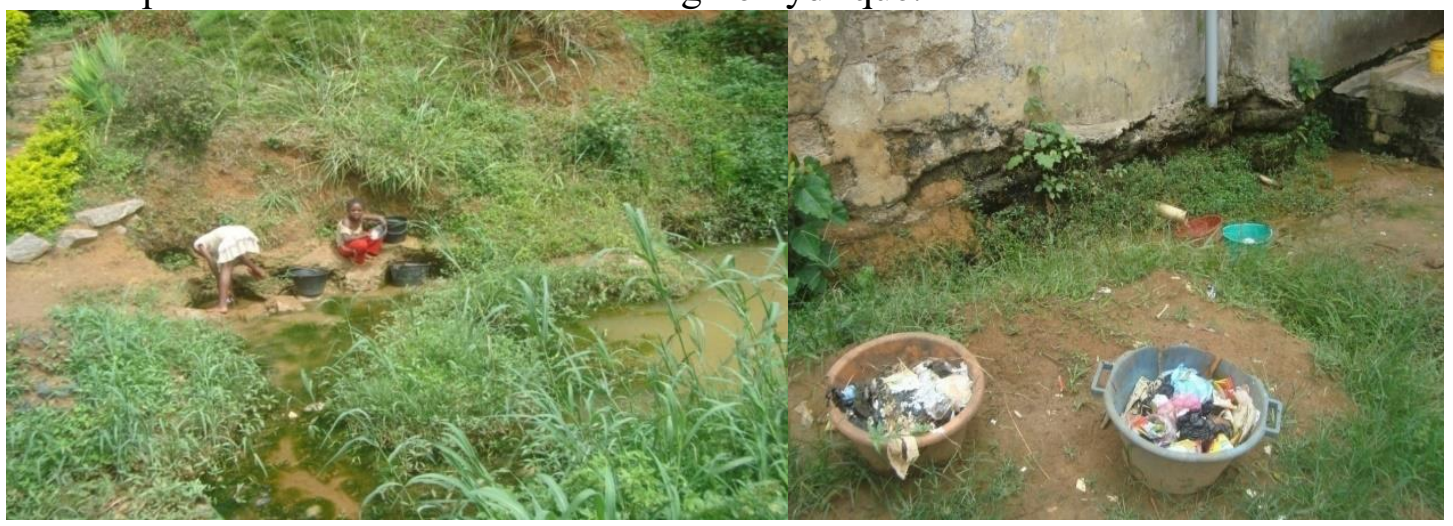

Photo 4: Source envahie par les herbes à Melen 9 (Cliché Kengmoé, Mai 2012)
Photo 5: Source non aménagée proche d'une latrine et des ordures ménagères Melen 9 (Cliché Kengmoé, Mai 2012) 
En somme, le bassin versant amont de l'Abiergué dispose de plusieurs points d'eau construits ou naturels. Les populations exploitent ces plans d'eau pour leur approvisionnement en eau afin de satisfaire leurs divers besoins ménagers.

\subsection{Analyse physico-chimique des eaux de la zone d'étude}

L'analyse des paramètres physico-chimiques des eaux de consommation a permis d'attester de la mauvaise qualité des eaux de source et de puits (Tableau 2).

En effet, les résultats des analyses physico-chimiques montrent que le pH des eaux de la source aménagée de Melen 6 et celui des eaux du puits amélioré de Melen 9, sont inférieurs à 6,14.

Cependant, le pH des eaux du puits sommaire de Melen 7A est de 6,56. Ces eaux ont une minéralisation faible. Même si les valeurs de conductivité des eaux échantillonnées sont de $272 \mu \mathrm{S} / \mathrm{cm}$ pour le puits amélioré de Melen 9 et de $594 \mu \mathrm{S} / \mathrm{Cm}$ pour le puits sommaire de Melen.

La conductivité de l'eau du puits amélioré de Melen 6 est estimée à $185,2 \mu \mathrm{S} / \mathrm{cm}$. Les valeurs de la température des eaux échantillonnées sont légèrement élevées (comprises entre 27 et $27,2^{\circ} \mathrm{C}$ ).

Tableau 2: Récapitulatif des paramètres physico-chimiques des eaux de puits et de sources

\begin{tabular}{|c|c|c|c|c|}
\hline $\begin{array}{c}\text { Site de prélèvement des } \\
\text { échantillons d'eau }\end{array}$ & Température $\left({ }^{\circ} \mathbf{C}\right)$ & $\mathbf{p H}$ & $\begin{array}{c}\text { Conductivité } \\
(\boldsymbol{\mu S} / \mathbf{C m})\end{array}$ & Nitrates (mg/l) \\
\hline Source Aménagée Melen 6 & 27,2 & 6,12 & 185,2 & 3,0 \\
\hline Puits amélioré Melen 9 & 27 & 6,14 & 272 & 3,6 \\
\hline Puits sommaire Melen 7A & 27,2 & 6,56 & 594 & 5,3 \\
\hline
\end{tabular}

Source: Waste Water Research Unit, Faculté des Sciences, Université de Yaoundé I, 2013

Par ailleurs, les résultats obtenus mettent en exergue la présence des nitrates à de très faibles quantités dans les eaux de puits et de source étudiés. Ces valeurs sont de 3,6 mg/l pour le puits amélioré de Melen 9, de 5,3 mg/l pour le puits sommaire de Melen 7A et de 3,0 $\mathrm{mg} / \mathrm{l}$ pour la source aménagée de Melen 6.

\subsection{Analyse bactériologique des eaux de la zone d'étude}

Les résultats des analyses bactériologiques des eaux du bassin versant amont de l'Abiergué sont consignés dans le Tableau 3. Ces résultats indiquent que les puits et la source d'eau étudiés ont une concentration élevée en coliformes fécaux (332 UFC/100 ml au total).

Ces concentrations sont très élevées dans les eaux du puits sommaire de Melen 7A (237 UFC/100 ml au maximum). Par contre, dans les eaux du puits amélioré de Melen 9 et celles de la source aménagée Melen 6, elles sont moyennes avec respectivement $57 \mathrm{UFC} / 100 \mathrm{ml}$ au maximum et $38 \mathrm{UFC} / 100$ $\mathrm{ml}$ au maximum. 
Par ailleurs, il ressort des résultats de laboratoire que les eaux de puits et de source étudiées ont une forte concentration en streptocoques fécaux (Tableau 3). Les concentrations de ces germes pathogènes sont plus élevées dans les eaux de source aménagée (11 UFC/100 ml au maximum) de Melen 6. Elles sont faibles dans le puits sommaire de Melen 7A (6 UFC/100 ml au maximum) et le puits amélioré de Melen 9 (9 UFC/100 ml au maximum).

Tableau 3: Résultats des analyses bactériologiques des eaux de puits et source

\begin{tabular}{|l|c|c|}
\hline $\begin{array}{c}\text { Site de prélèvement des } \\
\text { échantillons d'eau }\end{array}$ & $\begin{array}{c}\text { Coliforme Fécaux (UFC/100 } \\
\text { ml) }\end{array}$ & $\begin{array}{c}\text { Streptocoques Fécaux } \\
\text { (UFC/100 ml) }\end{array}$ \\
\hline Source aménagée Melen 6 & 38 & 11 \\
\hline Puits amélioré Melen 9 & 57 & 9 \\
\hline Puits sommaire Melen 7A & 237 & 6 \\
\hline
\end{tabular}

Source : Waste Water Research Unit, Faculté des Sciences, Université de Yaoundé I, 2013

\subsection{Risques sanitaires pour les populations du bassin versant amont de l'Abiergué}

Dans le bassin versant amont de l'Abiergué, les populations sont probablement exposées à divers risques sanitaires liés à la mauvaise qualité des eaux qu'elles consomment.

En effet, sur le terrain, les latrines sont construites le plus souvent à proximité des puits et d'autres points d'eau, pourtant exploités par les ménages pour leurs activités quotidiennes.

Aussi, les eaux pluviales en s'écoulant entrainent tous les déchets des toilettes vers les points d'eau mal aménagés ou non aménagés. Par conséquent, l'eau captée est de qualité douteuse.

De plus, la nappe phréatique de notre zone d'étude affleure, surtout dans les bas-fonds du quartier. Cette situation favorise aussi la contamination de l'eau des puits ou des sources par les lixiviats et autres déchets organiques issus des latrines et des fosses septiques.

Enfin, l'absence d'égouts et la présence des latrines à fond perdu sont aussi des facteurs qui contribuent à la pollution des nappes phréatiques. Les eaux usées évacuées dans les rigoles aménagées devant les maisons ou sur les parcelles, constituent un facteur supplémentaire de contamination des cours d'eau et de la nappe phréatique dans la zone étudiée.

En saison pluvieuse, les eaux de puits, de sources et de rivières sont majoritairement polluées par les déchets solides. Cette période favorise le transport des polluants, de matériaux divers par les torrents qui les déposent dans les points d'eau, en l'occurrence les sources et les rivières. Certains ménages déversent immédiatement les ordures ménagères dans les rivières. Cette pratique est courante dans les bas-fonds, où circule la rivière Edjoa Mballa. En période des pluies, les eaux usées en contact avec les tas d'ordures ménagères en putréfaction contaminent la nappe phréatique par infiltration. L'agencement spatial des points d'approvisionnement en eau, des latrines et 
des dépotoirs d'ordures a permis de mettre en évidence un risque élevé de contamination des eaux de surface et souterraines (Figure 4).

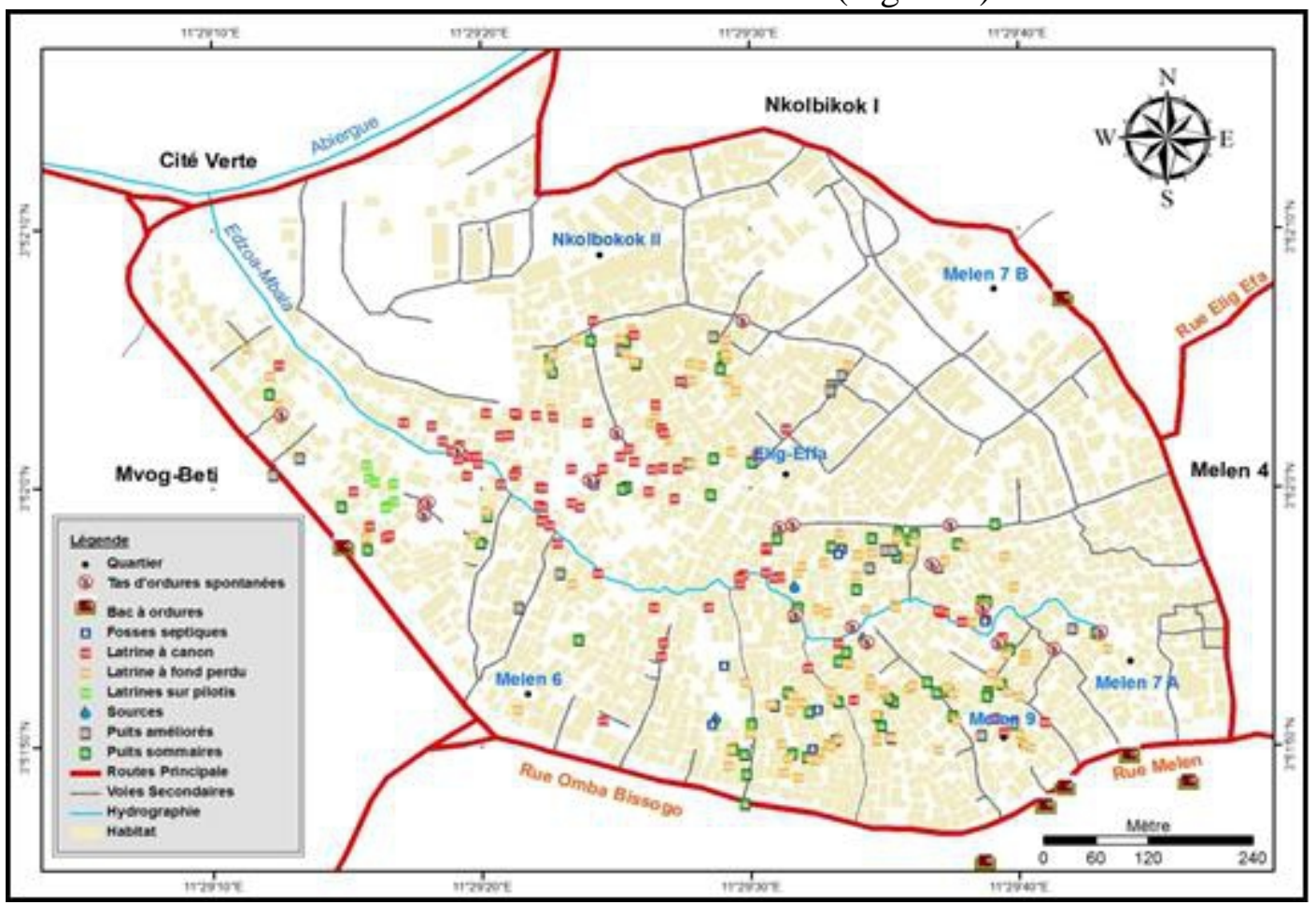

Figure 4 : Répartition des points d'eau, latrines et dépotoirs d'ordures dans la zone d'étude (Source : Levées GPS, 2013)

En outre, la promiscuité dans la zone d'étude accentue la pollution, puisque le point d'eau, même aménagé devant la maison, reste toujours susceptible de recevoir, par le système de vase communiquant, les microbes et autres agents pathogènes, en provenance des latrines ou des fosses septiques voisines.

Cependant, la stagnation des eaux usées liée à l'inexistence de systèmes fiables d'évacuation des eaux (ex : les égouts), constitue un danger pour l'environnement immédiat et une menace pour la santé des habitants de cette zone d'étude.

En effet, les eaux usées, quelles que soient leur provenance, sont susceptibles de contaminer les sources d'eau environnantes. L'infiltration de ces eaux et des eaux vannes peut contaminer la nappe phréatique, et par ricochet, la ressource en eau. Les eaux souterraines sont également contaminées par lixiviation.

La figure 5 montre l'évolution dans le temps des maladies liées à l'eau dans notre zone d'étude entre 2016 et 2018. En effet, dans le bassin versant amont de l'Abiergué, le nombre de malades enregistrés au «Centre de Santé 
Privé Espoir de Melen 9 » et souffrant des maladies hydriques, a évolué en dents de scie au cours de ces dernières années (Figure 5), mais avec une tendance globale à la baisse (sauf pour la fièvre typhoïde).

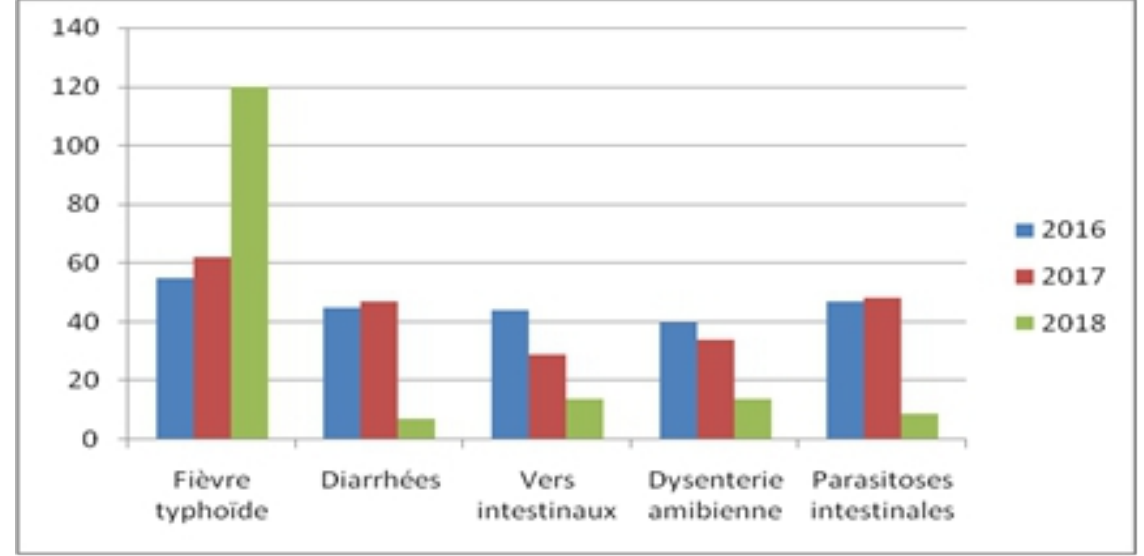

Figure 5: Évolution des cas de maladies hydriques entre 2016 et 2018 au «Centre de Santé Privé Espoir de Melen 9».

Source : Compilation du registre de consultation du Centre de Santé Privé Espoir de Melen 9 en 2019.

Parmi ces maladies, la fièvre typhoïde est la plus fréquente des pathologies diagnostiquées avec 237 cas enregistrés au Centre de Santé Privé Espoir de Melen 9 entre 2016 et 2018. À l'inverse des autres maladies, la fièvre typhoïde a connu plutôt une évolution croissante en passant de 57 cas de malades en 2016 à 60 cas en 2017 et 120 cas en 2018. Pour les diarrhées, 42 cas ont été enregistrés en 2016, puis 44 cas en 2017 et 13 autres cas en 2018.

Le nombre de personnes atteintes de vers intestinaux a baissé entre 2016 et 2018 avec notamment, 41 cas en 2016, 29 cas en 2017 et 14 cas en 2018. De même, la dysenterie amibienne a connu une évolution décroissante entre 2016 et 2018: 40 malades enregistrés en 2016 et 17 cas en 2018. Concernant les parasitoses intestinales, prés de 45 cas ont été recensés en 2016, 46 en 2017 et 8 en 2018.

\section{Discussion}

Cette étude a permis de montrer que la plupart des habitants du bassin versant de l'Abiergué utilise les eaux des sources $(n=7)$, des puits $(n=136)$ ou des bornes fontaines $(\mathrm{n}=8)$. Mais, ces ouvrages hydrauliques sont à proximité des latrines, des ordures ménagères et des eaux usées susceptibles de les polluer. Cette proximité entre les puits et les latrines a été signalée par Mougoué et al. (2012) dans la ville de Yaoundé. Ces auteurs ont rapporté que cet etat de fait favorise le transfert latéral et vertical des coliformes et des streptocoques fécaux souillant à la fois la nappe souterraine et les eaux 
superficielles. Parallèlement à ce qui précède, l'étude conduite par Menye (2018) sur la dégradation du cadre de vie des populations de Douala V a montré que la présence des déchets solides et liquides est à l'origine de la pollution des eaux de puits et de sources. Cet auteur a affirmé que la prédominance des latrines sommaires à fond perdu et leur proximité avec les puits, seraient les principales causes de la pollution des eaux souterraines et des maladies hydriques. Le même constat a été fait par Eyong (2019) dans la ville de Bafoussam.

Les résultats des analyses physico-chimiques des eaux utilisées par les populations de cette zone d'étude ont révélé que le $\mathrm{pH}$ des eaux de la source aménagée de Melen 6 et celui des eaux du puits amélioré de Melen 9, sont inférieurs à 6,14. Ces valeurs ne sont pas conformes à la norme de potabilité du Cameroun. Cependant, le $\mathrm{pH}$ des eaux du puits sommaire de Melen 7A $(\mathrm{pH}=6,56)$ respecte les seuils admis par l'OMS, la France et le Cameroun.

Par ailleurs, ces eaux ont une minéralisation faible. Même si les valeurs de la conductivité des eaux échantillonnées qui sont de $272 \mu \mathrm{S} / \mathrm{cm}$ pour le puits amélioré de Melen 9 et de $594 \mu \mathrm{S} / \mathrm{Cm}$ pour le puits sommaire de Melen 7A respectent la norme camerounaise de 2008, strictement inférieure à $1000 \mu \mathrm{S} / \mathrm{cm}$ elles sont largement au-dessus de la valeur de conductivité de l'eau recommandée par l'OMS (strictement inférieure à $250 \mu \mathrm{S} / \mathrm{cm}$ ). La conductivité de l'eau du puits amélioré de Melen $6(185,2 \mu \mathrm{S} / \mathrm{cm})$ respecte cette norme de l'OMS. Les valeurs de température des eaux échantillonnées sont légèrement élevées (comprises entre 27 et 27,2 ${ }^{\circ} \mathrm{C}$ ). Celles-ci sont audessus de la norme française qui est inférieure à $25^{\circ} \mathrm{C}$. Par ailleurs, les résultats des analyses d'eau ont mis en exergue la présence des nitrates en faibles proportions. Ils sont actuellement sans danger pour les populations dans les eaux de puits et de source étudiés car leurs taux sont largement inférieurs aux normes recommandées par la France $(\leq 50 \mathrm{mg} / \mathrm{l})$, par l'OMS $(<44 \mathrm{mg} / \mathrm{l})$ et par le Cameroun ( $\leq 50 \mathrm{mg} / \mathrm{l})$.

$\mathrm{Au}$ regard de tous les résultats concernant les caractéristiques physicochimiques des eaux de puits et de source étudiées, il ressort que ces eaux sont impropres à la consommation et peuvent nuire à la santé humaine. D'ailleurs, Talom (2009) a montré que dans les quartiers précaires de Yaoundé, il existe différents types de toilettes, à savoir les latrines traditionnelles à fond perdu et les latrines à canon, qui sont généralement très polluantes et contaminent les ressources en eau.

Par ailleurs, les ana lyses bactériologiques des eaux prélevées dans la zone d'étude ont révélé que les puits et la source étudiés ont une concentration importante en coliformes fécaux. Les concentrations de ces germes pathogènes sont plus élevées dans les eaux du puits sommaire de Melen 7A (237 UFC/100 ml au maximum) que dans les eaux du puits amélioré de Melen 9 (57 UFC/100 ml au maximum) et la source aménagée (38 UFC/100 ml au 
maximum) de Melen 6. S'agissant des streptocoques fécaux, il ressort que leur concentration a été plus élevée dans les eaux de source aménagée (11 UFC/100 $\mathrm{ml}$ au maximum) de Melen 6. Ces des streptocoques ont été plus faibles dans le puits sommaire de Melen 7A (6 UFC/100 ml au maximum) et le puits amélioré de Melen 9 ( 9 UFC/100 $\mathrm{ml}$ au maximum).

La présence des coliformes fécaux et des streptocoques fécaux dans une eau la rend automatiquement impropre à la consommation humaine au regard des normes de potabilité de l'eau recommandées par la France, l'OMS et le Cameroun. Les travaux menés par Manfo (2017) sur l'accès à l'eau potable dans la Commune de Yaoundé $6^{\text {ème }}$ corroborent nos résultats. En effet, cet auteur a indiqué que les caractéristiques physico-chimiques (température, conductivité, nitrate, ammonium, $\mathrm{PH}$ et les ions chlores) et bactériologiques (streptocoques et coliformes fécaux) des eaux des puits et des sources de cette région ne respectent pas les normes de potabilité et cela exposent les populations locales aux maladies hydriques.

Cette situation a été aussi notée par Bopda (2018), qui soutient que les populations des quartiers précaires de la ville de Yaoundé sont exposées aux maladies hydriques essentiellement liées à la forte présence des coliformes et streptocoques fécaux dans les eaux de puits et de sources. Kengne (2018) dans le même ordre d'idée, souligne que les eaux de puits et de sources dans les quartiers carrière et Madagascar à Yaoundé présentent une forte concentration en coliformes et streptocoques fécaux; cela vulnérabilise la santé des populations riveraines.

La pollution de ces eaux pourrait être liée à de nombreux facteurs, notamment l'urbanisation anarchique dans le bassin versant Amont de l'Abiergué et les mauvaises conditions d'hygiène et d'assainissement (prédominance des latrines sommaires à fond perdu, eaux usées et déchets solides divers). Ces facteurs seraient à l'origine de la précarisation des conditions de vie des populations et la pollution des eaux de puits et sources. D'ailleurs, Kengne (2000) a indiqué que les mauvaises gestion des déchets solides et liquides entraîne des problèmes de santé pour les populations et l'insalubrité dans les villes. Assako Assako et al. (2006) renchérissent en montrant que la concentration humaine, dans les espaces limités des villes présente de réels et préoccupants risques sanitaires du fait de la multiplication des quartiers précaires, d'une pauvreté urbaine quasi-généralisée associées à l'insuffisance des systèmes d'assainissement, de gestion des déchets d'où une pollution des ressources en eau.

De même, Wethe et al. (2003) ont rapporté que la multiplication des maladies hydriques dans les villes africaines est accentuée par la pollution des ressources en eau. Ces eaux véhiculent une multitude de germes pathogènes pour l'homme et se rendent responsables de nombreuses épidémies. 


\section{Conclusion}

La santé des populations est étroitement liée à la qualité des eaux consommées et du cadre de vie. L'étude des risques sanitaires liés à l'approvisionnement en eau dans le bassin versant amont de l'Abiergué à Yaoundé a permis de relever l'existence de plusieurs modes d'approvisionnement en eau par les populations dans le bassin versant amont de l'Abiergué, notamment le réseau de distribution de la Camwater pour les personnes nanties et, les puits, les sources aménagées ou non et les bornes fontaines payantes pour les populations pauvres.

La mauvaise gestion des déchets solides et liquides des ménages, la prédominance des latrines sommaires à fond perdu situées à proximité des puits et des sources contribuent à la contamination des eaux de ces ouvrages. Les analyses physico-chimiques et bactériologiques menées dans différents points d'approvisionnement (source aménagée, puits sommaire, puits aménagé) ont permis de noter que les eaux de la zone d'étude ont des conductivités dépassant les seuiles autorisés par l'OMS. Ces eaux sont aussi fortement contaminées par les germes pathogènes (coliformes et streptocoques fécaux). Ces ressources hydriques utilisées par les populations sont donc impropres à la consommation. La mauvaise qualité de celles-ci expose les populations à divers risques sanitaires dont les maladies d'origine hydriques telles que les diarrhées, la fièvre typhoïde, les dysenteries amibiennes et les parasitoses intestinales.

\section{References:}

1. Assako Assako R.J, Djilo Tonmeu C.A, et Bley D., 2006, Gestion des eaux usées et des déchets domestiques en relation avec le risque sanitaire à Kribi : une approche géo-anthropologique, communication présentée aux XVIIIe journées scientifiques de la Société d'Ecologie Humaine, Marseille, 23 p.

2. Bopda T., 2018, Acteurs locaux et préservation du cadre de vie des populations de la ville de Yaoundé, Mémoire de Master, IPD, 130 p.

3. Conférence de Dublin, 1992, Conférence internationale sur l'eau et l'environnement :le développement dans la perspective du $21^{\mathrm{e}} \mathrm{S}$; Rapport, 57 p.

4. Eyong F.J. 2019, Accès à l'eau à Bafoussam et risque sanitaires, Mémoire de Master, Université de Yaoundé 1, 163 p.

5. Global Compact Network-France. https://www.globalcompactfrance.org/document/les.17 objectifs de développement-durable-et-leurs-169-cibles-89.

6. Kengmoé T.E., 2013, Pratiques d'hygiène, d'assainissement et impacts environnementaux et sanitaires dans le Bassin versant amont de l'Abiergué, Université de Yaoundé 1, Mémoire de Master, 150 p. 
7. Kengne F., 1992, Les problèmes environnementaux dans les grandes villes Camerounaises. In la situation de l'environnement au Cameroun, Yaoundé, Fondation Friedrich-Ebert, Imprimerie SaintPaul, pp. 7-25

8. Kengne N., 2000, Assainissement des eaux usées domestique par lagunage à macrophytes : cas de SIC-MAETUR à Biyem Assi II 5 (Yaoundé), rapport du colloque, $40 \mathrm{p}$.

9. Kengne P., 2018, Impact de l'assainissement individuel sur les eaux souterraines dans les quartiers Carrière et Madagascar à Yaoundé, Mémoire de Master, Université de Douala, 137 p.

10. Manfo Y., 2017, Défis contemporain d'accès à l'eau potable dans la Commune d'Arrondissement de Yaoundé $6^{\text {ème }}$, Mémoire de Master, Université de Yaoundé 1, 130 p.

11. Menye M., 2018, Assainissement des déchets solides, liquides et impacts sanitaires dans la Commune de Douala V, Mémoire de Master, Université de Douala, 160 p.

12. Mougoué B., Ngnikam E., Feumba R. 2012, Impacts sanitaires et environnementaux de l'assainissement des eaux usées et des excréta dans les quartiers précaires de Yaoundé (Cameroun), in Eau et Assainissement, Institut de l'Energie et de l'Environnement de la Francophonie (IEEF), numéro 92-2 ${ }^{\mathrm{e}}$ trimester: 60-64.

13. Mougoué B., Ngnikam E., Wanko A., Feumba R., Noumba I., 2012, Analysis of faecal sludge management in the cities of Douala and Yaoundé, in Cameroon SS-13, pp 11-21.

14. Ngambi J.R., 2008, Etude des indicateurs de pollution des ressources en eau et conséquences sanitaires dans la ville de Yaoundé: Le cas de l'arrondissement de Yaoundé $1^{\mathrm{er}}$, Mémoire de DEA de Géographie, Option Gestion des Ressources Naturelles, 115 p.

15. Ngnikam E., 2000, Evaluation environnementale et économique de système de gestion des déchets solides municipaux : analyse de cas de Yaoundé au Cameroun.. Thèse de Doctorat des sciences et techniques du déchet, LAEPSI/INSA de Lyon, France, 363 p.

16. Ngnikam E., Mougoué B., et Tietché F., 2007, Eau, Assainissement et impact sur la santé : étude de cas d'un écosystème urbain à Yaoundé, in Actes des JSIRAUF, Hanoi, 6-9 novembre 2007, 13 p.

17. Plan International, 2015, L'eau c'est la vie, journée mondiale de l'eau. En ligne https ://www.plan-international.fr.

18. Sanchoir et Bopda, 1998, Atlas réginal du Sud Cameroun 103 p.

19. Suchel J.B., 1987, Les climats du Cameroun, Thèse d'Etat Ph.D, Université de Bordeau III, France, 1188 p.

20. Talom S., 2009, «Une opportunité pour viabiliser les latrines », in la gazette du quartier $\mathrm{n}^{\circ} 17$, pp 14-15 
21. Tchako G., 2005, Approvisionnement en eau potable dans l'arrondissement de Yaoundé III : Le cas d'Ahala. Mémoire de Maîtrise. Université de Yaoundé I, 101p.

22. Wethé J., Radoux M., et Tanawa E., 2003, Assainissement des eaux usées et risques socio-sanitaires et environnementaux en zones d'habitat planifié de Yaoundé. Dossier: Santé et environnement, volume $4 \mathrm{n}^{\circ} 1$, mai. 